\title{
Wavelet-based multifractal analysis of nonlinear time series: The earthquake-driven tsunami of 27 February 2010 in Chile
}

\author{
B. A. Toledo* \\ Institute of Aeronautical Technology (ITA) and World Institute for Space Environment Research (WISER), CTA/ITA/IEFM, \\ São José dos Campos-SP 12228-900, Brazil and Departamento de Física, Facultad de Ciencias, Universidad de Chile, Las Palmeras 3425, \\ Nuñoa, P.O. Box 653, Santiago, Chile
}

A. C.-L. Chian

Observatoire de Paris, LESIA, CNRS, 92190 Meudon, France National Institute for Space Research (INPE) and World Institute for Space Environment Research (WISER), P.O. Box 515, 12227-010 São José dos Campos-SP, Brazil

\author{
E. L. Rempel \\ Institute of Aeronautical Technology (ITA) and World Institute for Space Environment Research (WISER), CTA/ITA/IEFM, \\ São José dos Campos-SP 12228-900, Brazil
}

R. A. Miranda

University of Brasilia (UnB), Faculty of Technology, Gama campus, and Plasma Physics Laboratory, Institute of Physics, Brasília-DF 70910-900, Brazil and Institute of Aeronautical Technology (ITA) and World Institute for Space Environment Research (WISER), CTA/ITA/IEFM, São José dos Campos-SP 12228-900, Brazil

P. R. Muñoz

Institute of Aeronautical Technology (ITA) and World Institute for Space Environment Research (WISER), CTA/ITA/IEFM, São José dos Campos-SP 12228-900, Brazil

J. A. Valdivia

Departamento de Física, Facultad de Ciencias, Universidad de Chile, Las Palmeras 3425, Ñuñoa, P.O. Box 653, Santiago, Chile (Received 6 September 2012; revised manuscript received 20 December 2012; published 28 February 2013)

\begin{abstract}
We study general multifractal properties of tidal gauge and long-wave time series which show a well defined transition between two states, as is the case of sea level when a tsunami arrives. We adopt a method based on discrete wavelets, called wavelet leaders, which has been successfully used in a wide range of applications from image analysis to biomedical signals. First, we analyze an empirical time series of tidal gauge from the tsunami event of 27 February 2010 in Chile. Then, we study a numerical solution of the driven-damped regularized long-wave equation (RLWE) which displays on-off intermittency. Both time series are characterized by a sudden change between two sharply distinct dynamical states. Our analysis suggests a correspondence between the preand post-tsunami states (ocean background) and the on state in the RLWE, and also between the tsunami state (disturbed ocean) and the off state in the RLWE. A qualitative similarity in their singularity spectra is observed, and since the RLWE is used to model shallow water dynamics, this result could imply an underlying dynamical similarity.
\end{abstract}

DOI: 10.1103/PhysRevE.87.022821

PACS number(s): 89.75.Fb, 47.53.+n, 91.30.Nw, 05.45.Tp

\section{INTRODUCTION}

Tsunamis are long waves that are generated by submarine earthquakes [1], landslides [2,3], volcanic eruptions [4], and atmospherical phenomena [5,6]. Tsunamis can result in extreme wave heights at the shore, while in the deep open ocean they can have extremely small amplitudes and move much faster $[7,8]$, which makes it very difficult to detect them throughout the ocean. Although the basic characteristics of a tsunami wave are quite clear $[7,8]$, its detailed behavior can become extremely complex due to its strong dependence on the local coastal topography [6,9-11]. This complex dynamics has resulted in many numerical approaches of increasing

*btoledo@fisica.ciencias.uchile.cl complexity [12-14], all of them solving some form of a nonlinear shallow water wave equation, which may be derived from depth integrating the Navier-Stokes equations or from first principles [15]. One commonly used nonlinear model of shallow water waves is the regularized long wave equation (RLWE) which has also been used to study some features of fluid turbulence or spatiotemporal chaos [16].

A successful tool for studying this kind of complex behavior is based on the wavelet transform and multifractal formalisms [17-21]. Multifractal formalisms were introduced in the context of fully-developed turbulence data analysis as a tool for studying the experimental observation of departures from the Kolmogorov theory (K41) on homogeneous and isotropic turbulence [17]. The first successful multifractal description of singular measures [22] was a statistical approach based on the continuous wavelet transform [23]. Multifractal wavelet 
methods have been applied to fully developed turbulence, econophysics, meteorology, physiology, and DNA sequences [24] to cite a few applications.

In this paper we use an approach based on discrete wavelet bases, namely, the wavelet leaders developed by Jaffard [18], to compare the scaling behavior of an inertial subrange estimated from the time series of an on-off intermittent solution [16] of the RLWE with a qualitatively equivalent inertial subrange estimated from the tidal gauge time series recorded at three different stations along the Chilean coast when the earthquake with a moment magnitude of $8.8 \mathrm{M}_{w}$ occurred in the central zone of Chile on 27 of February 2010 [25] (27F).

The paper is organized as follows. In Sec. II, we outline the multifractal formalism based on wavelet leaders. Next, in Sec. III, we describe the tidal gauge measurements used, the locations from where the data was collected, the method used to define our data time interval, and the results of applying the wavelet leaders multifractal analysis. In Sec. IV we study a spatiotemporally chaotic solution of the RLWE for a particular on-off transition and compare our results, in a qualitative manner, to the tsunami behavior according to their singularity spectra. And finally, in Sec. V we present the conclusions of our analysis.

\section{OUTLINE OF MULTIFRACTAL FORMALISM}

Let us consider a time series $x(t)$, and let us measure its local regularity around $t_{0}$ by its Hölder exponent $h\left(t_{0}\right) \geqslant 0$, which is defined as the largest $h$ such that

$$
\left|x(t)-P\left(t-t_{0}\right)\right| \leqslant C\left|t-t_{0}\right|^{h},
$$

where $P\left(t-t_{0}\right)$ is a polynomial of order $n$, such that $n<$ $h<n+1$ and $C>0$ [26]. Now, let us denote by $E_{h_{0}}$ the set of all points on the real line (the time series support) with a given Hölder regularity $h_{0}$, such that $1 \geqslant \operatorname{dim}\left(E_{h_{0}}\right) \equiv D\left(h_{0}\right)$ is the Hausdorff dimension of $E_{h_{0}}$. Since time series have finite resolution, it is not convenient to measure $D(h)$ directly, instead we resort to a multifractal formalism (MF) procedure. To set up the MF we will use the wavelet leaders approach [27] that has well established properties to analyze the multifractal spectrum of discrete time series. Let $\psi$ denote the mother wavelet and define

$$
\psi_{j, k}(t)=2^{-j / 2} \psi\left(2^{-j} t-k\right), \quad j, k \in \mathbb{Z},
$$

as the dilated and translated wavelets, which form an orthonormal basis of $L^{2}(\mathbb{R})$. Note that $k$ is the discretized time translation and $j$ is the time scale. The mother wavelet is characterized by the number of vanishing moments, which is defined by the positive integer $N_{\psi} \geqslant 1$ such that $\forall r=$ $0, \ldots, N_{\psi}-1$,

$$
\int_{\mathbb{R}} t^{r} \psi(t) d t=0
$$

and

$$
\int_{\mathbb{R}} t^{N_{\psi}} \psi(t) d t \neq 0 .
$$

Let

$$
d_{x}(j, k)=\int_{\mathbb{R}} x(t) \psi_{j, k}(t) d t
$$

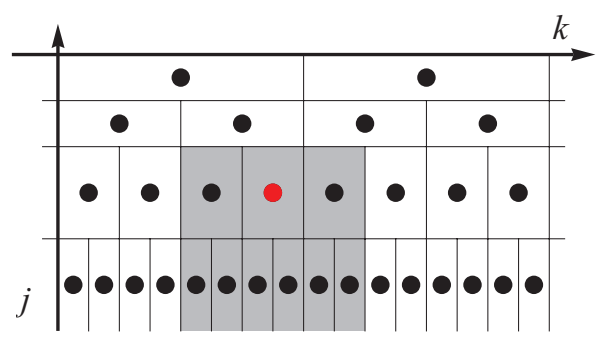

FIG. 1. (Color online) Space-scale plane and the dyadic tree. Discrete wavelet coefficients $d(j, k)$ are represented by dots $(\bullet)$ and the dyadic interval $\lambda_{j, k}$ by the surrounding rectangle. The shaded area sketches the subset $\Lambda_{j, k}$ associated with the wavelet leader $L_{x}(j, k)$ (red middle dot), adapted from Ref. [21].

denote the ( $L^{1}$-normalized) discrete wavelet transform coefficients of $x(t)$, where $j$ is related to the time scale $\tau=2^{j}$ and $k$ to the time translation $\left(t=2^{j} k\right)$. As defined in Ref. [21], the wavelet leaders $L_{x}(j, k)$ are multiresolution quantities defined as

$$
L_{x}(j, k)=\sup _{\lambda^{\prime} \subset \Lambda_{j, k} ; j^{\prime} \leqslant j}\left|d_{x}\left(\lambda^{\prime}\right)\right|
$$

where $\lambda^{\prime}=\lambda_{j^{\prime}, k^{\prime}}=\left[2^{j^{\prime}} k^{\prime}, 2^{j^{\prime}}\left(k^{\prime}+1\right)\left[\right.\right.$ and $\Lambda_{j, k}=\bigcup_{m \in\{-1,0,1\}}$ $\lambda_{j, k+m}$. The leader is defined along all the possible branches spawned by the $\Lambda_{j, k}$ interval at all higher resolutions $j^{\prime} \leqslant j$. Hence, for a given $k$ and $j$, the supremum is taken over the region shown in the schematic representation of Fig. 1.

For a fixed time scale $\tau=2^{j}$, the time averages of the $q$ th powers of the $L_{x}(j, k)$ are referred to as the structure functions

$$
S^{L}(j, q)=\frac{1}{n_{j}} \sum_{k=1}^{n_{j}} L_{x}(j, k)^{q},
$$

where $n_{j}$ is the number of leaders $L_{x}(j, k)$ available at scale $2^{j}$. It can be shown that

$$
S^{L}(j, q)=F_{q} 2^{j \zeta(q)}
$$

in the limit where $2^{j} \rightarrow 0$ [28], and $F_{q}$ is some constant. Parisi and Frisch [17,29] introduced the so-called multifractal formalism (MF), in which a Legendre transform establishes a link between $\zeta(q)$ and $D(h)$ as

$$
D(h)=1+\min _{q}[q h-\zeta(q)] .
$$

Therefore, the MF consists of obtaining an approximation of $D(h)$ from estimations of $\zeta(q)$ [21].

A last issue that should be remembered is the minimal Hölder regularity to apply the MF, which imposes the condition [30]

$$
h_{\min }=\liminf _{2^{j} \rightarrow 0}\left(\ln \sup _{k} \frac{\left|d_{x}(j, k)\right|}{\ln 2^{j}}\right)>0,
$$

which requires a bounded time series [26]. If this is not satisfied, a fractional integration has to be done [31]. Note that taking a derivative of order $\eta \in N$ is equivalent to multiplying the Fourier transform of the function by $(i \xi)^{\eta}$. Therefore, the inverse operator (integration of order $\eta$ ) requires the division of the Fourier transform by $(i \xi)^{\eta}$. This will produce a problem if the Fourier transform does not vanish at the origin, therefore, 
the alternative operator

$$
\widehat{I^{\eta}(f)}=\left(1+|\xi|^{2}\right)^{-\eta / 2} \hat{f}(\xi),
$$

where the hat $(\widehat{\ldots})$ denotes Fourier transform, is used, and which has the same behavior at high frequencies as that of the Riemann-Liouville fractional integral, but does not have the drawback at zero frequency and can be extended to noninteger values of $\eta$. This procedure shifts the Hölder exponents as

$$
h_{I^{\eta}(f)}\left(x_{0}\right)=h_{f}\left(x_{0}\right)+\eta, \quad \eta>-h_{\min },
$$

which produces a well defined leader scaling function as required [30,32].

The detailed derivation of the expressions recalled here, is beyond the scope of this study and the reader is referred to the books by Mallat [33] and Jaffard [18], and the citations in this section. For the numerical estimation of the multifractal quantities we used the MATLAB toolbox provided by Wendt et al. [28,30], which uses Daubechies wavelets [34].

\section{TIDAL GAUGE MEASUREMENTS}

The big earthquake of magnitude $8.8 \mathrm{M}_{w}$ that occurred on February 27 of 2010 at 06:34 (UTC) in the central zone of Chile was the result of the sudden displacement of the Nazca plate under the South American plate in an area that approximately extends from the peninsula of Arauco to the north of Pichilemu city, over an approximate rectangle of $450 \times 150 \mathrm{~km}^{2}$. The epicenter was located at $-36^{\circ} 17^{\prime} 23^{\prime \prime}$ (longitude) and $-73^{\circ} 14^{\prime} 20^{\prime \prime}$ (latitude) at a depth of $30.1 \mathrm{~km}$ [25]. The first informed tsunami wave was located in Pichilemu, at approximately 06:48 (UTC) [35], whereas in Valparaíso it was recorded at approximately 7:06 (UTC) [36].

The tidal gauge data we analyzed was obtained from the Sea Level Station Monitoring Facility [36], collected from bottom pressure sensors installed on Vaisala HydroMet MAWS110 equipment, with a sampling rate of $2 \mathrm{~min}$ (integrated time) and a sea level resolution of $\sim \pm 1.5 \mathrm{~mm}$ (deduced from the data). The 2 min sampling rate is contained in the time scale range associated with a tsunami, which starts at about 1 min [37]. Three Chilean stations were selected from north to south, located in the following places: Coquimbo, Valparaiso, and
Ancud, as shown in Fig. 2. These stations are maintained and operated by the Hydrographic and Oceanographic service of the Navy of Chile (SHOA) [38].

In what follows we designate as "background" the quiet preand post-tsunami oceanic behavior, for which the dynamics results mainly from the gravitational and Coriolis forcing, excluding other sources such as the atmospherical forcing. For the perturbed oceanic behavior, not coming from an atmospherical forcing but driven by the earthquake, we designate it as "tsunami." The leading tsunami wave (tsunami arrival) was approximately determined by observing the deviation from background tides in the tidal gauge records. To determine the time interval to analyze the tsunami wave, note that a tsunami introduces a strong perturbation in the statistical distribution of the data. On the other hand, we assume that the background is associated with a characteristic statistical distribution to compare with the tsunami state. This statistical characterization has some degree of variability due to the short lengths of our data sets, which is imposed by the sampling rate $(2 \mathrm{~min})$ and the tsunami time scale. Since the statistical moments of the increments are the structure functions of the time series [17,39], we begin by taking a time window of approximately 22 days, beginning at the tsunami arrival, and move it forward in time in steps of a day (720 points), resulting in 25 windows to analyze. Note that we take the background after the earthquake because the data has less gaps in that interval. According to Eq. (8) the function $\zeta(q)$ is related to the statistical moments of the time series [17]. Consequently, to obtain a qualitative measure of the range of variability of the statistical distribution of the time series, we can use the function $\zeta(q)$ for each data set contained in our moving window. In Fig. 3 we see the range of variability of $\zeta(q)$, which is related to the statistical distribution variability, after the tsunami arrival for the three locations. Note that it is possible to observe how the structure functions scaling exponents gradually converge, not to a single curve, but to a range of nonforced variability. For comparison we also show the probability density function (PDFs). In this way, we find the time shift which results in a value of $\zeta(q)$ that is in the range of nonforced variability, i.e., that excludes the tsunami.

From this calculation results that with more than seven days of elapsed time (this is not shown in Fig. 3), the tsunami effects

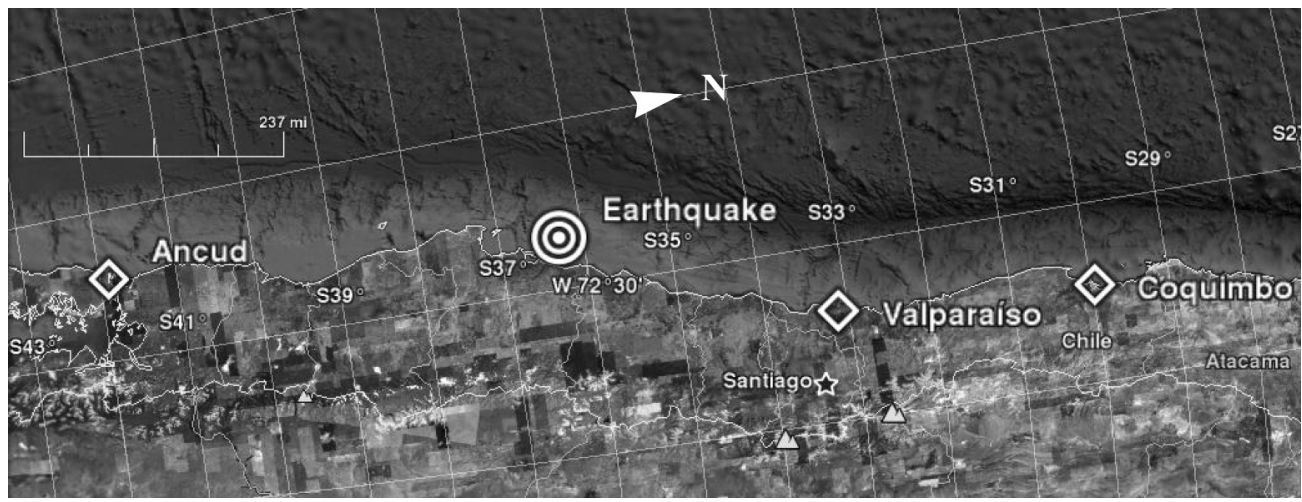

FIG. 2. Tidal gauge stations and the earthquake epicenter. The location of the tidal gauge stations is indicated with diamonds $(\diamond)$ and the epicenter of the earthquake is indicated with concentric circles ((@) ). This figure was taken from Google Earth. The epicenter was obtained from the Seismological Service of the University of Chile [25]. 

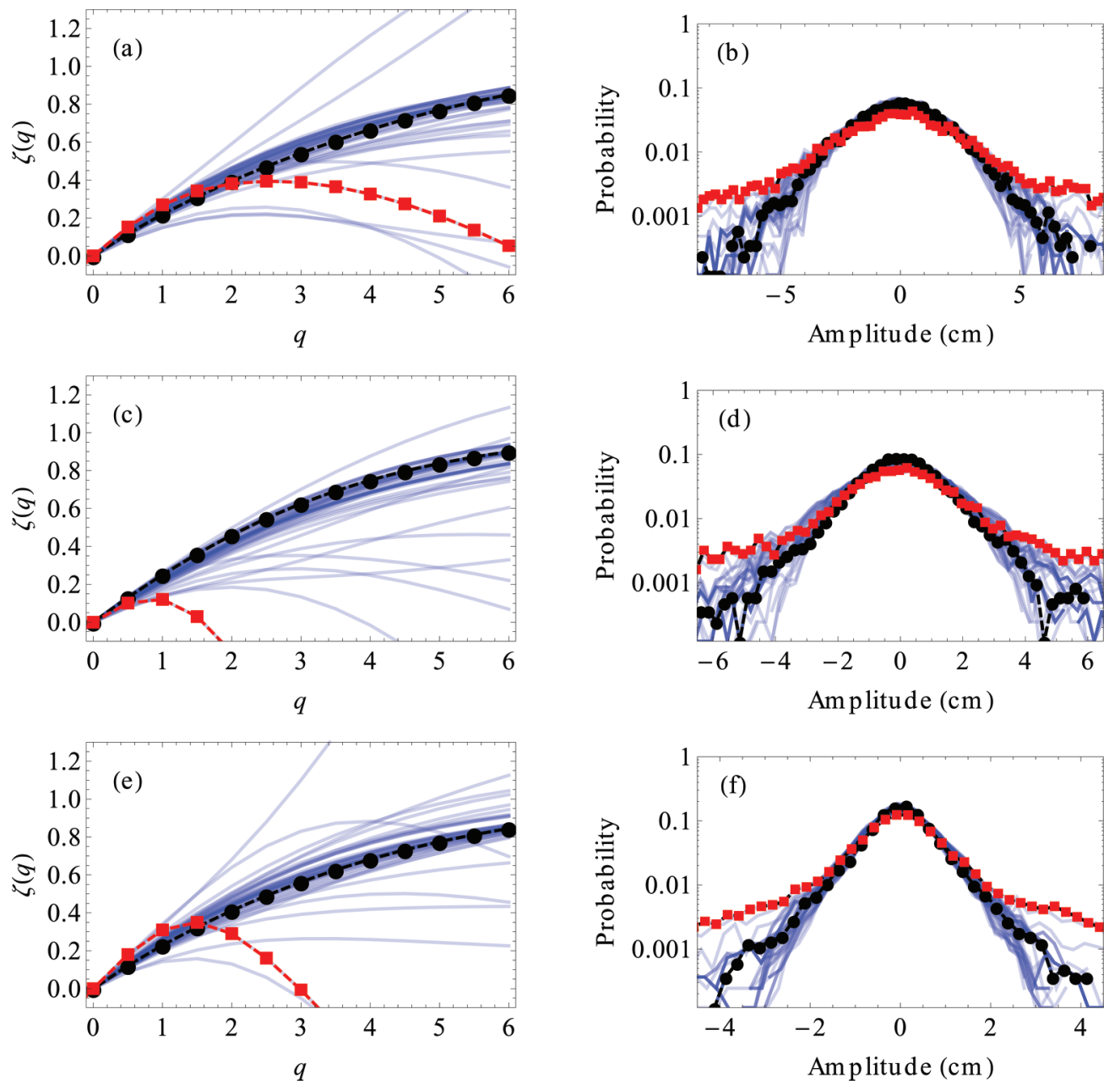

FIG. 3. (Color online) Range of variability of the structure function scaling exponent and the estimated PDFs. Observe the convergence zone for [(a),(b)] Coquimbo, [(c),(d)] Valparaíso, and [(e),(f)] Ancud as we move out from the tsunami arrival. The analyzed time series is associated with the black circle dashed line and the time series containing the tsunami is related to the red square dashed line.

are negligible, and from the dyadic restriction that imposes that our time series must have a length of $2^{n}$ points, we choose a length of 8192 points or $\approx 11$ days beginning at the tsunami arrival, a time interval that encompasses the tsunami perturbation. Note that this result depends on the geographic location.

Therefore, for the tsunami data we took points starting near the leading tsunami wave, after the earthquake [25], while for the background data we took a different starting point for each location in order to analyze the data of best quality, that is, with less gaps contained in the time window to be analyzed. Specifically, we start 14 days after the $27 \mathrm{~F}$ event for Coquimbo, 21 days after the 27F event for Valparaíso, and 16 days after the $27 \mathrm{~F}$ event for Ancud, all of them well beyond the "ringing" period [37]. We used approximately 11 days of data (8192 points) for both background and tsunami. The detide was done through the empirical mode decomposition (EMD) method [40,41], which is different from the empirical orthogonal function (EOF) expansion, also known as the principal component analysis or singular value decomposition method. The EMD method is built on the idea of identifying the various scales in the data that are used to characterize the multifractality; therefore, orthogonality is not a consideration, but scales are. Since orthogonal decomposition is a characteristic for linear systems, violating this restriction is not a shortcoming. By this method, the signal is decomposed in intrinsic mode functions (IMF) which may or may not be orthogonal. In our application, we extracted the tides by retaining the first three IMFs, corresponding to the high frequency range. Figure 4 shows the sum of them, a sample (four days) of the sea level measurements for each location, starting near the leading tsunami wave. Since the wavelet analysis needs a constant sampling rate, a few gaps (less than $2 \%$ ) in the data were filled with a linear interpolation. This interpolation should not affect our results because of two reasons: first, we used Daubechies wavelets with $N_{\psi}=33$ [42], therefore the linear part is taken out by the wavelet filtering as shown by Eq. (3), and second, the number of interpolated points is a small fraction of the total number of data points measured $(<2 \%)$. We computed the singularity spectrum $D(h)$ of the background time series using a mother wavelet with an increasing number of null moments, from $N_{\psi}=3$ to $N_{\psi}=40$. Around $N_{\psi}=33$ we obtained a nearly invariant singularity spectrum estimation (this remnant variability is expected to exist due to the nonstationarity of the dynamics). We use this value for the rest of the paper. 


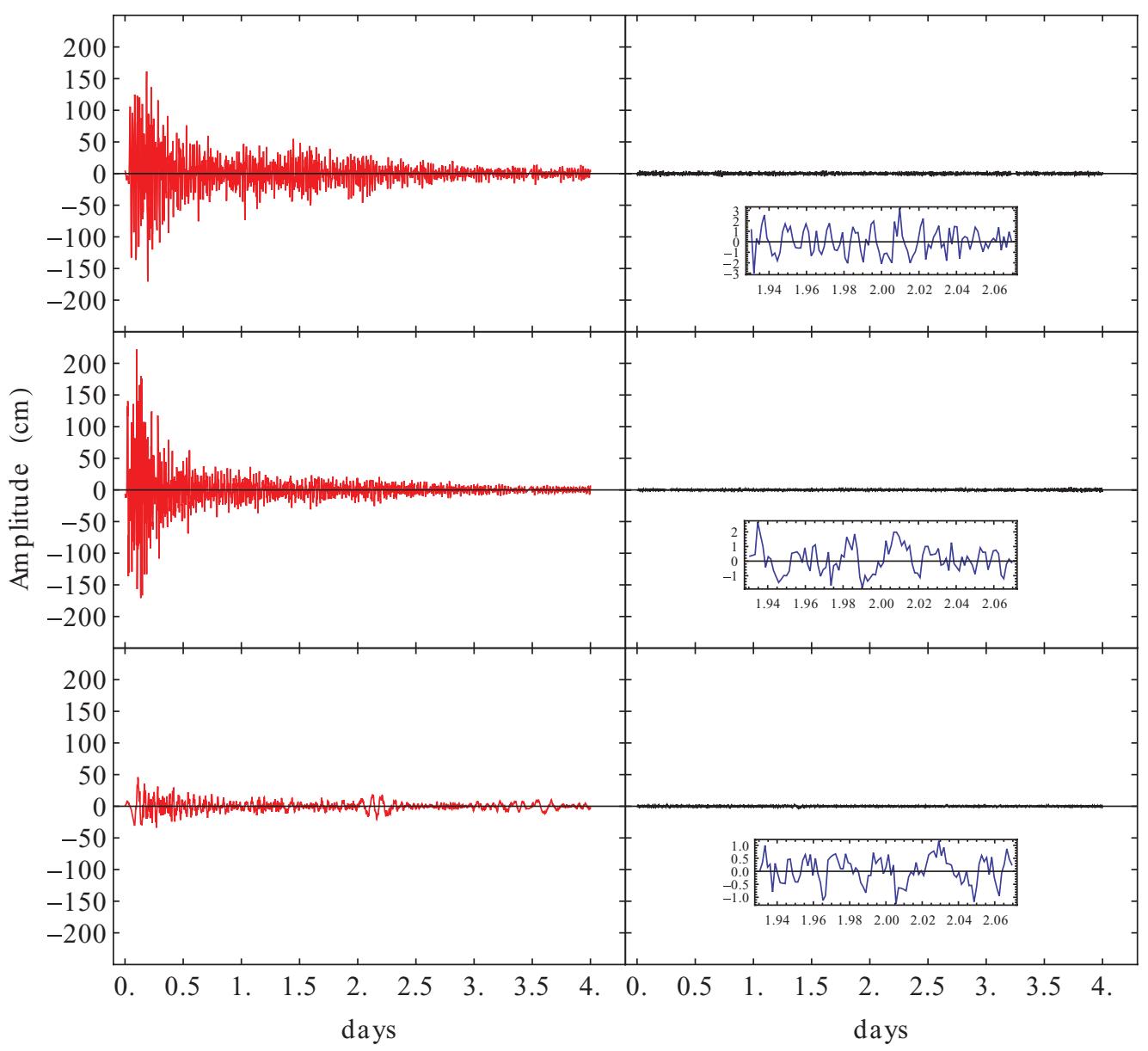

FIG. 4. (Color online) The detied tidal gauge measurements used in this study. From top to bottom we show Coquimbo, Valparaíso, and Ancud. A black line represents the background in the right panels and a red line represents the tsunami in the left panels. The insets show a zoom of the background around day 2 .

From Fig. 4 it is readily apparent the large impact of tsunami waves when compared to the background behavior. However, the background also possesses a complex dynamics that includes the tides, the Coriolis forcing (inducing geostrophic flow), and the atmospherical forcing $[43,44]$. Those combined effects produce in some locations Fourier spectra with as many as 390 modes [43], with fractal properties [45-47]. Therefore, we can apply the MF to extract part of its statistical properties and observe how its multifractality changes under the tsunami forcing. We searched for scaling behaviors in the wavelet leaders calculated from the time series [28], as shown in Fig. 5, where the selected scaling region [Eq. (8)] is shown for both background and tsunami, according to the measurements at the three stations and the procedure to define them as described above. As established by Eq. (10), we need $h_{m}>0$, however, a direct calculation for the tsunami time series gives $h_{m}<0$ for all three stations. For Coquimbo we obtained $h_{m} \approx-0.316$, for Valparaíso $h_{m} \approx-0.366$, and for Ancud $h_{m} \approx-0.271$. Therefore, we apply the minimal order fractional integration with $\eta>-h_{m}$ such that we obtain a spectrum $\zeta_{\eta}(q)$ that produces a $D_{\eta}(h)$ with $h_{m}>0$. This value of $\eta$ is used to construct $\zeta(q)=\zeta_{\eta}(q)-q \eta$ and $D(h)$. After the fractional integration of a given order $\eta$ for each site, the alignment region [48] (scaling region) persisted.
Even though other scaling regions were found, we chose the range 8-32 min which is inside the characteristic scaling region of tsunami waves (1-100 min) [37], and it was consistently observed for all three locations, in both background and tsunami time series.

In Fig. 6 we show the scaling exponents $\zeta(q)$ deduced from Eq. (8), for background and tsunami, at all three stations. The range of $q$ values used was obtained from the empirical criterion described in Ref. [49] (compare with the wavelet linearization criteria [50]). The nonlinearity of these curves is related to intermittency, which usually is associated with a departure from Gaussianity or monofractality [17], represented by a straight line. Therefore, both background and tsunami are intermittent according to their scaling exponents. The tsunami data displays a stronger intermittency, as shown for $q>0$, which focuses on large amplitude fluctuations. The intermittency in the background data means that extreme events are always present in the ocean and the tsunami forcing leads to a statistical enhancement of these extreme events. Also, note that the intermittency during the tsunami is strongly dependent on the location, while for the background it does not seem to change appreciably. This highlights the role of the local topography and bathymetry, which contribute with their own resonances to tsunami events and in general to seiches and harbor oscillations [37]. 

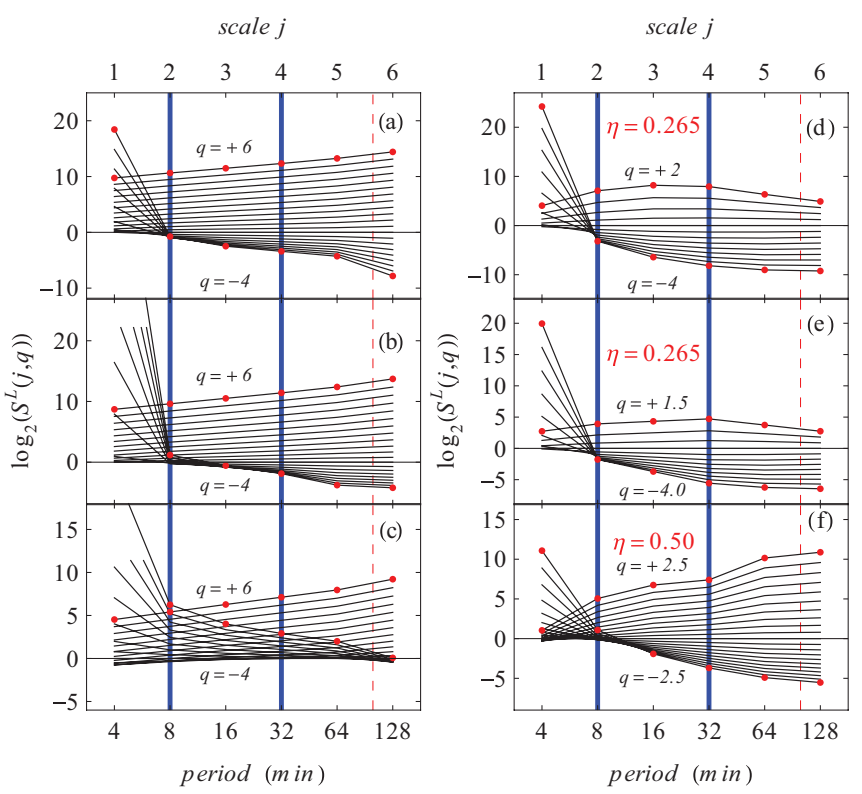

FIG. 5. (Color online) Scaling region (alignment region [48]) selected in this study, for background (left) and tsunami (right). The upper panel corresponds to Coquimbo, the middle panel to Valparaíso, and the bottom one to Ancud. The scaling region is between the two thick blue lines and the red dashed line shows the upper bound for tsunami spectra ( $\approx 100 \mathrm{~min}$ ). The order $\eta$ of the fractional integration for tsunami is also shown, which is not required for background.

Finally in Fig. 7 we show the estimated singularity spectrum $D(h)$ [Eq. (9)] for each location, for both background (black dots) and tsunami (red squares). The change in $D(h)$ from background to tsunami is readily apparent. After the tsunami arrives the singularity spectrum of the ocean has an enlarged range of Hölder exponents, which is associated with the emergence of new fractal sets [20], related to the wave dynamics. We also observe a dependence on location, as expected. Figure 7 shows that the singularity spectrum $D(h)$ of the tsunami extends over $h<0$, which suggests that the signal slope is locally not bounded. Another interesting feature is suggested by the singularity spectrum associated with Ancud, which possesses the most complex dynamics of the three locations. As shown in Fig. 7(c), it has a tendency to $D(h)<0$ in the right section of the spectrum; this nontrivial behavior may be related to models of random fractals [51,52] and will be further analyzed elsewhere.

Although the physical picture responsible for the observed tsunami dynamics is not well understood at present, we know that the unboundedness of certain quantities is not uncommon in physical models. For example in the context of fully developed turbulence an unbounded velocity may be the result of the compressibility of the fluid [17]. In our context, we could expect that the negative values for the Hölder regularity may have to do with the highly complex sea surface behavior associated with dynamical processes excited by the tsunami energy injection. For example, it is known that the air entrainment produced by the wave breaking [53], which is increased during a tsunami event, may change the water compressibility in the mixing layer [54]. If the void fraction is increased by the air entrainment, then the sound

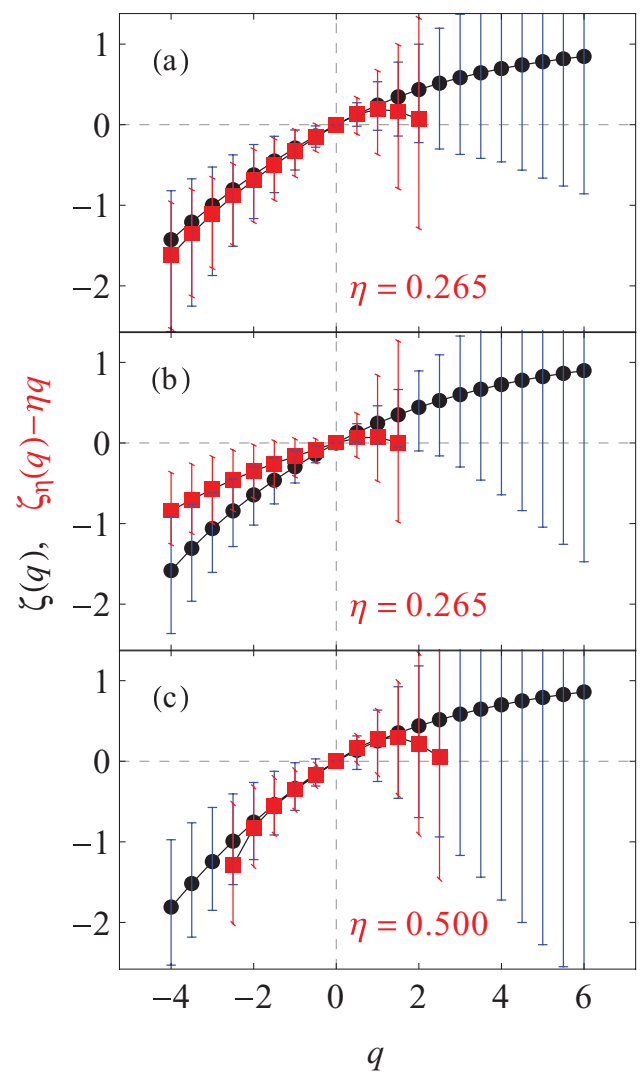

FIG. 6. (Color online) Structure function scaling exponents corresponding to tidal gauge measurements in Fig. 4, in the same order: (a) Coquimbo, (b) Valparaíso, and (c) Ancud. For tsunami we used red squares, and for background, black circles. The order $\eta$ of the fractional integration is also shown. Bootstrap error bars are included.

speed in water can become smaller than the sound speed in air [55], which could make water at the wave surface even more turbulent [56]. Hence, this chain of events could suggest an ingredient contributing to the highly complex sea surface dynamics associated with values of $D(h)$ for $h<0$, obtained by our analysis, in the tsunami data.

Now we will analyze the meaning of $h<0$ by an independent approach. As already shown [20], this is due to jumps in the signal, which are also a well known characteristic of shock waves. This last issue was noted by Salem et al. [57] when they analyzed the properties of the ambient solar wind, without distinguishing between slow and fast streams. The resulting structure functions are a consequence of a statistical mix of slow and fast wind properties, including the rapidly changing boundaries between slow and fast wind and interplanetary shocks. As in our case, their structure function scaling exponents curve down for increasing $q$ (Fig. 2 in Ref. [57]) and this, when applying a Legendre transformation, results in a singularity spectrum $D(h)$ defined for $h<0$. We follow their procedure to show that by removing the large amplitude tsunami waves, we obtain a nondecreasing function $\zeta(q)$. Since this procedure involves a filtering on the amplitude, we have to remove the background tides and other nonlinear waves, whose amplitude variations are similar in amplitude or height to the tsunami, and this time, instead of splitting the signal in IMFs, we use the usual harmonic 


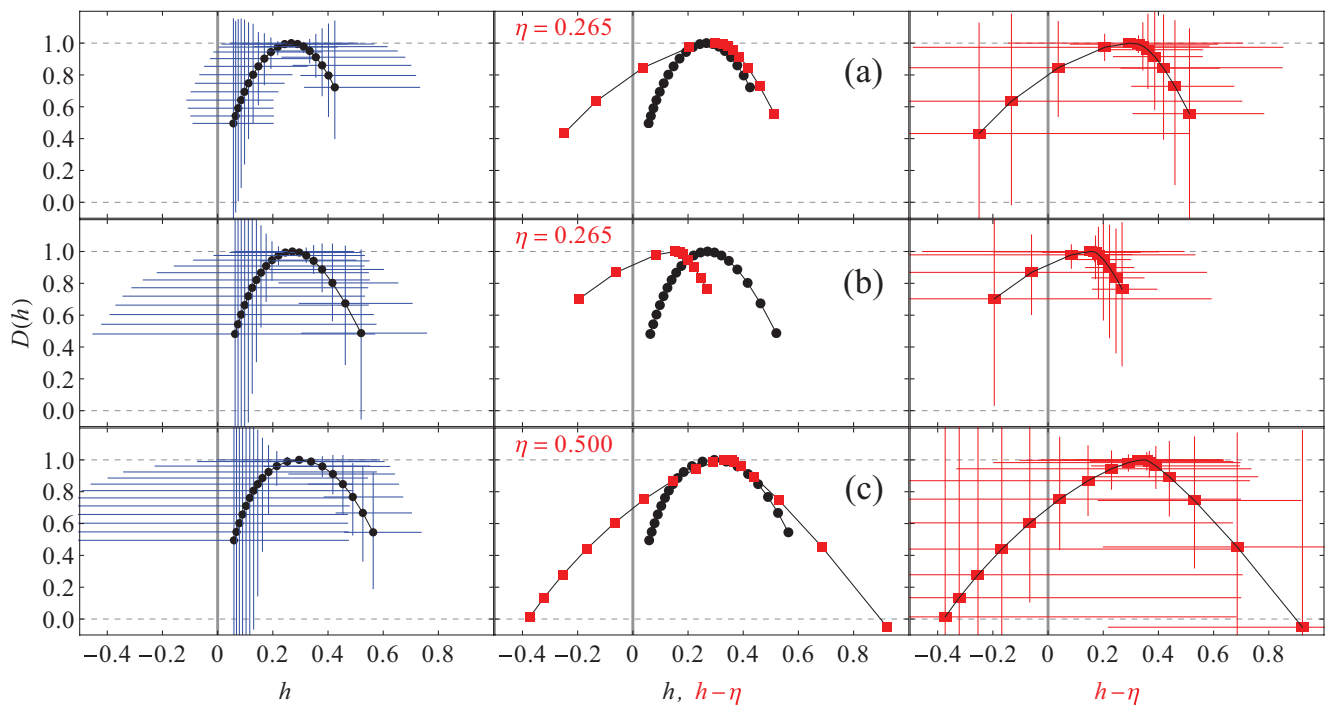

FIG. 7. (Color online) Singularity spectra corresponding to tidal measurements in Fig. 4, in the same order: row (a) Coquimbo, row (b) Valparaíso, and row (c) Ancud. For tsunami we used red squares, and for background, black dots. Note that for tsunami, $D(h)$ is defined for $h<0$. The order $\eta$ of the fractional integration is also shown. Left and right columns show the error estimated with a bootstrap [28]. The thick gray line shows the divide between background and tsunami at $h=0$.

analysis [43]. For removing the large amplitude waves, we used Daubechies wavelets and following Salem et al. [57], we define a scale-dependent threshold $\eta_{j}$,

$$
\eta_{j}^{2}=F\left\langle\left|d_{x}(j, k)\right|^{2}\right\rangle_{k}=F \sigma_{j}^{2},
$$

where $\sigma_{j}^{2}$ is the variance at scale $j$ calculated over all points $k$. The conditioning factor $F$ is a constant, independent of scale, arbitrarily chosen, with the only restriction that not too many points should be excluded by the conditioning. Thus, we consider the background component as characterized by $\left|d_{x}(j, k)^{2}\right|<\eta_{j}^{2}$, while the tsunami component is defined by $\left|d_{x}(j, k)^{2}\right| \geqslant \eta_{j}^{2}$.

Figure 8(a) shows the detided tsunami time series for Valparaíso, where we see that the maximum wave height was a little over $2 \mathrm{~m}$. Figure 8(b) shows the result after the wavelet filtering defined by Eq. (13), for the estimated tsunami [red (light gray)] and background (black), in both cases using Daubechies wavelets with $N_{\psi}=10$ and $F=0.1$. Figure 8(c) shows the structure function scaling exponents estimated for the measured background (black dots) and ten curves from the filtering procedure with $F=0.1 \times i$, for $i=1, \ldots, 10$. No value of $F$ reproduced the estimated measured background $\zeta(q)$ function from the tsunami filtering.

This result leads us to two conclusions. First, after removing most of the large amplitude waves, the structure function scaling exponent $\zeta(q)$ becomes a nondecreasing function of $q$. Therefore, after applying the Legendre transformation, there will be no values of $D(h)$ for $h<0$. Thus, a singularity spectrum $D(h)$ defined for $h<0$ is a characteristic of a tsunami (more specifically, of a signal with an envelope resembling that of a tsunami). And second, this estimated background does not behave statistically as the measured background. To understand this, recall that we have arbitrarily given a recipe to extract the tsunami waves, which is a strong energy injection mechanism. Therefore we should expect the energy distribution in this region to be different from the background. When we have a tsunami at the
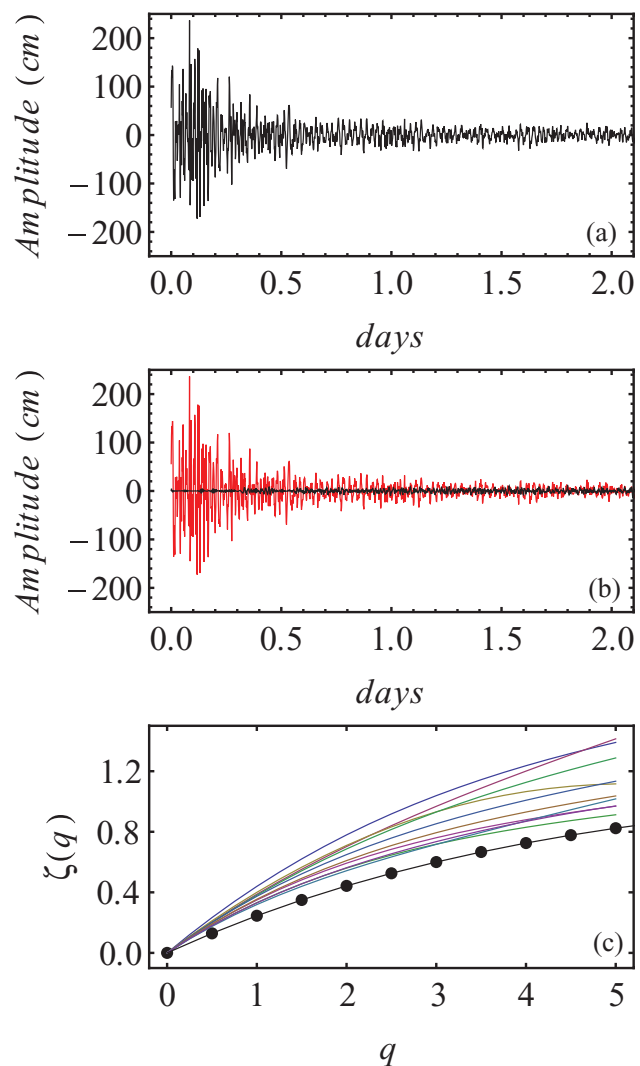

FIG. 8. (Color online) Tsunami filtering for Valparaíso. (a) Detided tsunami time series; (b) the filtering according to amplitude variance produces an estimation for background (black line oscillating around zero amplitude) and tsunami; and (c) structure function scaling exponents $\zeta(q)$ for estimated backgrounds and measured background in black dots. 


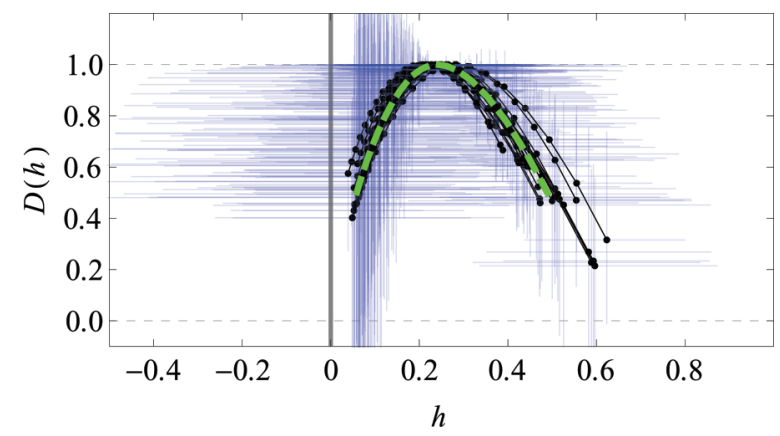

FIG. 9. (Color online) Several singularity spectra for background at Valparaíso. We took 21 time series of about 11 days, with exactly one nonoverlapping day. Five of them in the last part of March of 2010 and 16 in January of 2010, with 8192 points each. As shown, the estimation of $D(h)$ to the left of its maximum, which is associated with $q>0$, is very stable. The thick green dashed line represents the mean value. The parameters used were $N_{\psi}=33, j \in[2,4]$, and $q \in[-4,6]$. Bootstrap error bars are included.

measurement point, the tsunami energy cascades from large to small scales, inducing wave amplitude perturbations similar to those commonly seen in the background state, however, our calculations suggest that their statistics are different for all wave amplitude perturbations. This means that we cannot completely remove the perturbation in the statistical wave height distribution, introduced by the tsunami forcing, using only Eq. (13). Hence, this is a completely nonlinear system in which the small and large amplitudes are intrinsically coupled by the turbulent energy cascade, related to its multifractality.

A last issue regarding the data analysis is the robustness of our estimation. In Fig. 7 we showed the bootstrap estimated error (or confidence intervals) for the singularity spectrum estimation. Since a tsunami is a unique event, this technique provides valuable information. However, the background for each location can be regarded as a dynamic state of low statistical variability for time periods not including strong atmospherical forcing, as suggested by Fig. 3. Since this is our case in a temporal neighborhood of the tsunami event, we can obtain several estimations of $D(h)$ for the background. This will provide us with a more accurate estimation for the bounds of variability of $D(h)$ for the background. In Fig. 9 we show the result after taking 21 time series, each containing 8192 points, 16 time series before the tsunami and 5 after the tsunami and beyond the ringing period. As shown, the estimated singularity spectrum is very stable, especially in the region to the left of the maximum, where in the case of the tsunami a range of $h<0$ would appear. This is an additional test to the statistical significance of the estimations shown in Fig. 7.

\section{SOLUTIONS OF THE REGULARIZED LONG-WAVE EQUATION}

Since the RLWE basically describes the dynamics of shallow water long waves, it is tempting to associate the intermittent behavior observed in this model to the backgroundtsunami transition observed in the previous section. In this section we will describe the on-off intermittent states for a particular solution of the RLWE through the MF to compare with the results of the previous section.

The RLWE was proposed by Peregrine [58] and Benjamin [59] as an alternative to the Korteweg-de Vries (KdV) equation, since some mathematical properties of the $\mathrm{KdV}$ equation, such as the existence and stability of solutions, are difficult to study. Later, He [60] derived this same equation as a model for nonlinear drift waves in plasmas with an $\mathrm{ad}$ hoc periodic driving and damping terms in order to study the transition to chaos. Therefore, the driven-damped RLWE is given by Ref. [60]

$\partial_{t} \phi+c \partial_{x} \phi+f \phi \partial_{x} \phi+a \partial_{t x x} \phi=-v \phi-\epsilon \sin (x-\Omega t)$,

where $a, c$, and $f$ are constants, $\nu$ is a damping parameter, $\epsilon$ is the driver amplitude, and $\Omega$ is the driver frequency. Originally, Peregrine [58] obtained Eq. (14) without the two terms on the right-hand side from the momentum equation for the mean horizontal velocity of water elevation $\phi(x, t)$ of an irrotational flow by assuming that waves only travel in one direction and the ratios between wave amplitude and water depth and between water depth and wavelength are small. Here, wavelength means the distance in which significant changes in surface height occur. The third-order derivative term in Eq. (14) expresses the effect of the vertical acceleration of water on pressure.

Following Rempel et al. [16], we define periodic boundary conditions $\phi(x, t)=\phi(x+2 \pi, t)$ and solve numerically with $a=-0.28711, c=1, f=-6, v=0.1$, and $\Omega=0.65$. These parameter values can be chosen arbitrarily with the exception of $a$, which must be negative for physical reasons and to avoid numerical instability [60]. Here, the values are chosen in order to qualitatively model the statistical properties of a tsunami wave with an intermittent behavior previously studied in the RLWE $[61,62]$. Thus we fix the driver amplitude $\epsilon=0.2001$. For the details of the pseudospectral numerical solution see Ref. [16]. A typical example of an on-off intermittency described by this equation is shown in Fig. 10, where we show the amplitude evolution for a fixed point in space, $\phi\left(x_{0}, t\right)$, with $x_{0} \approx 1.885$.

The on state is characterized by a temporally chaotic and spatially regular behavior, while the off state is characterized

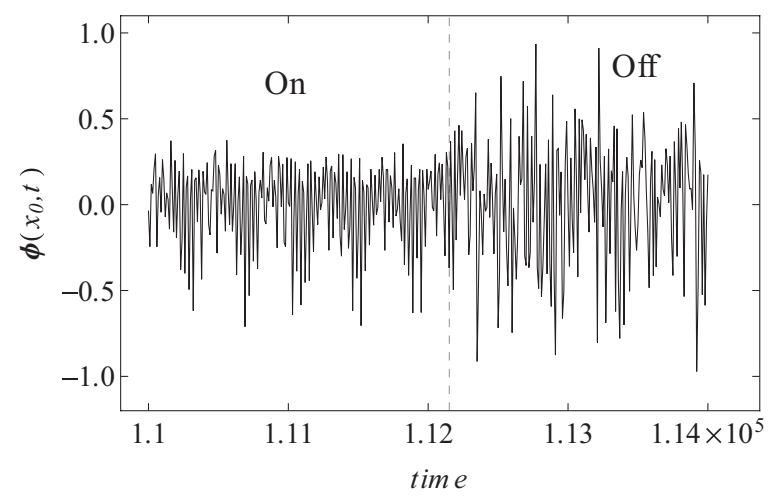

FIG. 10. On-off intermittent solution of the driven-damped RLWE, showing the behavior of $\phi\left(x_{0}, t\right)$, with $x_{0} \approx 1.885$. The solution alternates randomly between on and off states. Here we show one of those on-off transitions. 


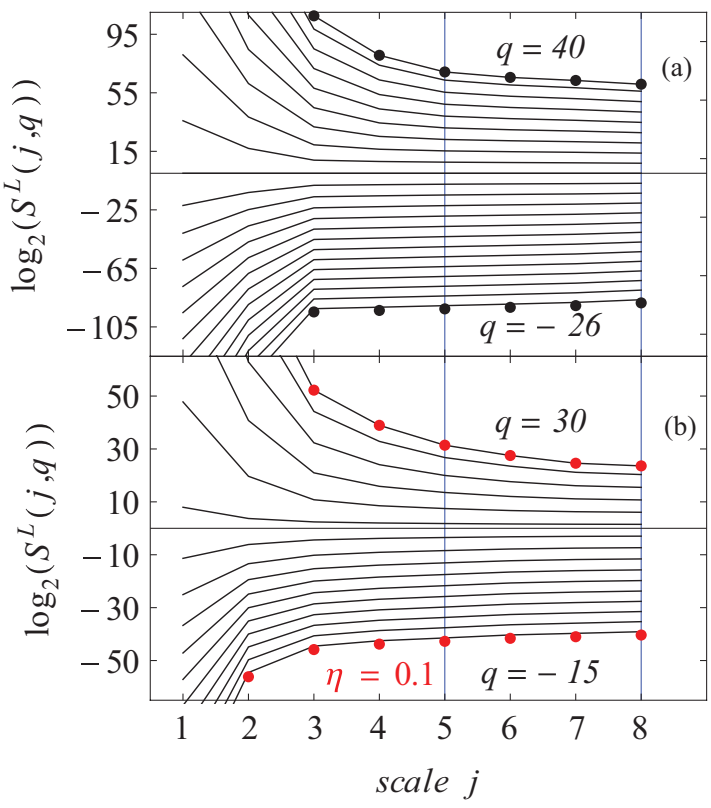

FIG. 11. (Color online) Logarithm of structure functions vs scale $j$, for on state (a) and off state (b) of the RLWE. Note the scaling region from $j=5$ to $j=8$.

by spatiotemporally chaotic patterns. The transition from spatial regularity to spatial irregularity is due to an attractorwidening crisis that occurs after the collision of the spatially regular attractor with an unstable saddle orbit, a behavior described in a series of papers [63-65]. It is important to recall at this point that we are not modeling the tsunami wave behavior realistically as it approaches the coast. Nevertheless, the statistical properties of the tsunami wave are similar to this particular solution of the RLWE, which again suggests a similar dynamical origin for those statistical behaviors, as we will substantiate below. For each state (on/off) we observe different scaling behavior as exemplified in Fig. 11, which was obtained using Daubechies wavelets with $N_{\psi}=21$ [42] since the intermittent solution is very smooth at short time scales.
Hence we are interested in the fractal-like behavior at larger time scales.

From the scaling analysis shown in Fig. 11, we obtain Fig. 12. Figure 12(a) shows intermittency for the range of scales selected and that intermittent behavior increases from on to off states [17]. For the off state we also followed the empirical criteria of Ref. [49]. This result can be interpreted in terms of multifractality as shown in Fig. 12(b) where we estimate the singularity spectrum $D(h)$ [19], which gives approximately the dimension of the set of points in the time series that locally scale as $\sim 2^{j h\left(t_{0}\right)}$ [21]. First, we observe that $D(h)$ shows a change in the sets that bound and define the dynamics, and also the emergence of new ones. This is a behavior expected according to Chian et al. [62]. The increase in the range for $h$ corresponds to the emergence of new fractal sets from the on to the off state. Second, from the definition of the Hölder regularity [18] we know that increasing values of $h$ correspond to a more regular behavior. Therefore, Fig. 12(b) shows that the main component of the off state is less regular than the main component of the on state. We know that in the off state the amplitude changes more chaotically than in the on state, which is consistent with the transition from temporal chaos to spatiotemporal chaos. Recall that the RLWE belongs to a class of equations whose solutions may develop the phenomenon known as wave overturning or wave breaking [66], where the derivative of the wave profile becomes undefined. In our case, the wavelet filtering captures a set of points that behaves locally as jumps at coarse scales, although at finer scales our solution does not show jumps nor wave overturning. We want to emphasize that this is not an artifact of the procedure since any natural phenomena (what we want to approximate) resembling fractality is strongly dependent on a particular range of scales, in our case the coarse scales [67].

Since the RLWE basically describes the dynamics of shallow water waves, it is tempting to associate the on-off transition with the background-tsunami transition since their related detailed statistics behave in a similar manner as we have shown above. As suggested in our modeling, the tsunami state could be associated with the topological change and/or the
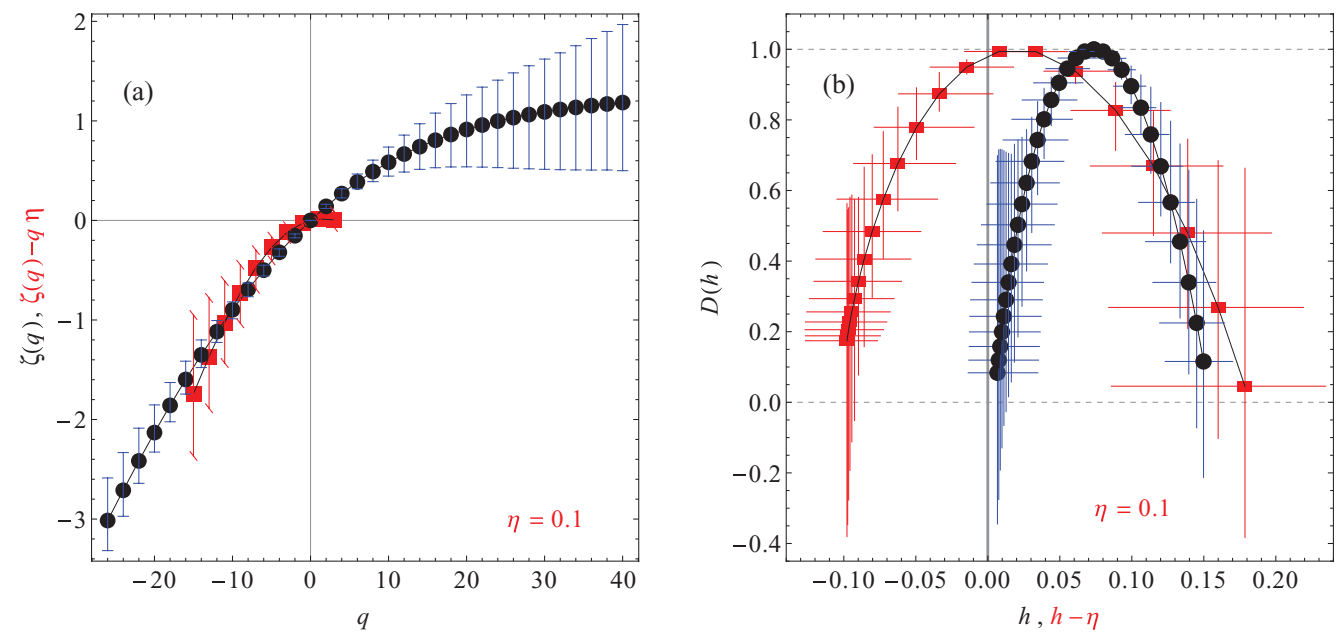

FIG. 12. (Color online) Multifractal analysis of the RLWE, On state in black circles and off state in red squares. (a) Scaling exponents $\zeta(q)$ derived from $S(j, q)$ and (b) singularity spectrum $D(h)$, derived from $\zeta(q)$, and corrected by fractional integration of order $\eta=0.1$. Note the negative range for the Hölder exponent $h$ for the off state. Bootstrap error bars are included. 
emergence of complex high dimensional (in the phase space) dynamical structures as suggested by Rempel et al. [16], which would drive the dynamics of the flow and therefore the surface wave behavior that we observe as sea level variation in the way described by $D(h)$. Although much more research is needed to corroborate this hypothesis, our results suggest a relation between this model for shallow water waves and the sea level variations caused by a tsunami.

\section{v. CONCLUSIONS}

We studied the impact of a tsunami arrival on the multifractal behavior of the ocean, for the coastal region of Chile, impacted by the earthquake of 27 February 2010. We concluded that the pre- and post-tsunami quiet background states can be regarded as of low statistical variability, for the three selected locations, over a time scale of two to three months (quiet atmospheric conditions were observed). We also observe a strong change associated with the tsunami arrival, with the most striking characteristic being the appearance of a multifractal subset for $h<0$ in the singularity spectrum $D(h)$, which could be related to the presence of shocks [57].

Regarding the RLWE model, although being onedimensional and solved with periodic boundary conditions, it still shows a behavior that is qualitatively similar to a tsunami. This suggests that from the viewpoint of dynamical systems, the underlying cause, assumed the same in both cases, is very robust. Let us remember that the RLWE was introduced as a mathematical model for the unidirectional propagation of long waves in systems that manifest nonlinear and dispersive effects [59], therefore, we believe our results justify to some extent its usage. In this scenario, the tsunami state could be associated with the topological change and/or the emergence of complex high dimensional (in the phase space) dynamical structures as suggested by Chian et al. [62] and Rempel et al. [16], which would drive the dynamics of the flow and therefore the surface wave behavior that we observe as sea level variations in the way described by $D(h)$.

\section{ACKNOWLEDGMENTS}

We thank anonymous referees for helpful suggestions and comments. This work was supported by FAPESP Grant No. 2010/09405-5. E.L.R acknowledges support from CNPq Grant No. 301634/2011-4 and FAPESP Grant No. 2011/507144. P.R.M. acknowledges support from FAPESP Grant No. 2011/10466-1. R.A.M. acknowledges support from FAPESP Grant No. 2010/00214-2. A.C.L.C. is grateful for the support of a Marie Curie International Incoming Fellowship and the hospitality of Paris Observatory. J.A.V. and B.A.T. are thankful for the financial support of Fondecyt Grants No. 1110135 and No. 1130273, respectively.
[1] W. C. Dragani, E. E. D’Onofrio, W. Grismeyer, and M. E. Fiore, Cont. Shelf Res. 26, 1543 (2006).

[2] E. A. Kulikov, A. B. Rabinovich, R. E. Thomson, and B. D. Bornhold, J. Geophys. Res., [Oceans] 101, 6609 (1996).

[3] I. V. Fine, A. B. Rabinovich, B. D. Bornhold, R. E. Thomson, and E. A. Kulikov, Mar. Geol. 215, 45 (2005).

[4] A. Belousov, B. Voight, M. Belousova, and Y. Muravyev, Pure Appl. Geophys. 157, 1135 (2000).

[5] S. Monserrat, I. Vilibic, and A. B. Rabinovich, Nat. Hazards Earth Syst. Sci. 6, 1035 (2006).

[6] A. B. Rabinovich and S. Monserrat, Nat. Hazards 13, 55 (1996).

[7] G. Margaritondo, Eur. J. Phys. 26, 401 (2005).

[8] O. Helene and M. T. Yamashita, Eur. J. Phys. 27, 855 (2006).

[9] A. B. Rabinovich, J. Geophys. Res. 102, 12663 (1997).

[10] T. Saito and T. Furumura, Geophys. J. Int. 177, 958 (2009).

[11] T. Saito, Geophys. J. Int. 182, 1515 (2010).

[12] V. V. Titov and C. E. Synolakis, J. Waterway, Port, Coastal, Ocean Eng. 124, 157 (1998).

[13] L. Tang, V. V. Titov, and C. D. Chamberlin, J. Geophys. Res., [Oceans] 114, C12025 (2009).

[14] I. K. Nikolos and A. I. Delis, Comput. Methods Appl. Mech. Eng. 198, 3723 (2009).

[15] L. D. Landau and E. M. Lifshitz, Fluid Mechanics (Course of Theoretical Physics), 2nd ed. (Butterworth-Heinemann, Oxford, 1987), Vol. 6.

[16] E. L. Rempel, R. A. Miranda, and A. C.-L. Chian, Phys. Fluids 21, 074105 (2009).

[17] U. Frisch, Turbulence: The Legacy of A. N. Kolmogorov (Cambridge University Press, Cambridge, 1996).

[18] S. Jaffard, Y. Meyer, and R. D. Ryan, Wavelets, Tools for Science and Technology, revised ed. (SIAM, Philadelphia, 2001).
[19] J. F. Muzy, E. Bacry, and A. Arneodo, Phys. Rev. Lett. 67, 3515 (1991).

[20] J. F. Muzy, E. Bacry, and A. Arneodo, Phys. Rev. E 47, 875 (1993).

[21] B. Lashermes, S. G. Roux, P. Abry, and S. Jaffard, Eur. Phys. J. B 61, 201 (2008).

[22] M. J. Feigenbaum, M. H. Jensen, and I. Procaccia, Phys. Rev. Lett. 57, 1503 (1986).

[23] A. Arneodo, E. Bacry, and J. F. Muzy, Physica A 213, 232 (1995).

[24] A. Bunde, J. Kropp, and H. J. Schellnhuber, in The Science of Disasters: Climate Disruptions, Heart Attacks, and Market Crashes, Physics and Astronomy Vol. 2, online library (Springer, Berlin, 2002).

[25] http://ssn.dgf.uchile.cl/.

[26] P. Abry, H. Wendt, S. Jaffard, H. Helgason, P. Goncalves, E. Pereira, C. Gharib, P. Gaucherand, and M. Doret, in Proceedings of the 32nd Annual International IEEE EMBS Conference, Buenos Aires, Argentina, 2010 (IEEE, New York, 2010).

[27] S. Jaffard, B. Lashermes, and P. Abry, in Wavelet Leaders in Multifractal Analysis, edited by T. Qian, M. Vai, and Y. Xu, Wavelet Analysis and Applications, Applied and Numerical Harmonic Analysis (Birkhäuser Basel, 2007), pp. 201-246, http://link.springer.com/chapter/10.1007\%2F9783-7643-7778-6_17.

[28] H. Wendt, P. Abry, and S. Jaffard, IEEE Signal Process Mag. 24, 38 (2007).

[29] S. Jaffard, J. Math. Pures Appl. 79, 525 (2000).

[30] H. Wendt, S. G. Roux, P. Abry, and S. Jaffard, Signal Process. 89, 1100 (2009). 
[31] H. M. Srivastava and R. K. Saxena, Appl. Math. Comput. 118 1 (2001).

[32] P. Abry, S. Jaffard, S. Roux, B. Vedel, and H. Wendt, in Wavelet Decomposition of Measures: Application to Multifractal Analysis of Images, edited by J. Byrnes, Unexploded Ordnance Detection and Mitigation, NATO Science for Peace and Security Series B: Physics and Biophysics (Springer, Netherlands, 2009).

[33] S. Mallat. A Wavelet Tour of Signal Processing (Wavelet Analysis \& Its Applications), 2nd ed. (Academic Press, San Diego, 1999).

[34] I. Daubechies, Ten Lectures on Wavelets (Society for Industrial and Applied Mathematics, Philadelphia, 1992).

[35] Armada de Chile, http://www.armada.cl/prontus_armada/site/ edic/base/port/inicio.html.

[36] Sea Level Station Monitoring Facility, UNESCO-IOC, http://www.ioc-sealevelmonitoring.org/index.php.

[37] Y. C. Kim, Handbook of Coastal and Ocean Engineering (World Scientific, Singapore, 2009).

[38] Servicio Hidrográfico y Oceanográfico de la Armada de Chile (SHOA), http://www.shoa.cl/index.htm.

[39] P. J. Brockwell and R. A. Davis. Introduction to Time Series and Forecasting, 2nd ed. (Springer, New York, 2002).

[40] N. E. Huang, Z. Shen, S. R. Long, M. C. Wu, H. H. Shih, Q. Zheng, N.-C. Yen, C. C. Tung, and H. H. Liu, Proc. R. Soc. London, Ser. A 454, 903 (1998).

[41] G. Rilling, P. Flandrin, and P. Gonçalvès, in Proceedings of the Sixth IEEE/EURASIP Workshop on Nonlinear Signal and Image Processing (NSIP '03), Grado, Italy, 2003 (IEEE, New York, 2003).

[42] I. Daubechies, Commun. Pure Appl. Math. 41, 909 (1988).

[43] J. Wright, A. Colling, D. Park, and O. U. O. C. Team, in Waves, Tides, and Shallow-Water Processes, Oceanography (Butterworth-Heinemann, in association with the Open University, 1999), http://www.sciencedirect.com /science/book/9780080363721.

[44] J. Pedlosky, Geophysical Fluid Dynamics (Springer, New York, 1987).

[45] J. L. Lions, R. Temam, and S. H. Wang, Nonlinearity 5, 1007 (1992).
[46] P. Yiou, D. Sornette, and M. Ghil, Physica D 142, 254 (2000).

[47] K. V. Koshel' and S. V. Prants, Phys. Usp. 49, 1151 (2006).

[48] P. Abry, P. Flandrin, M. S. Taqqu, and D. Veitch, Wavelets for the Analysis, Estimation, and Synthesis of Scaling Data (Wiley, New York, 2002), pp. 39-88.

[49] T. Dudok de Wit, Phys. Rev. E 70, 055302 (2004).

[50] B. Lashermes, P. Abry, and P. Chainais, Int. J. Wavelets, Multiresolut. Inf. Process. 02, 497 (2004).

[51] M. H. Jensen, G. Paladin, and A. Vulpiani, Phys. Rev. E 50, 4352 (1994).

[52] B. B. Mandelbrot and M. Frame, Fractals 17, 1 (2009).

[53] G. B. Deane and M. D. Stokes, Nature (London) 418, 839 (2002).

[54] O. University, in Seawater: Its Composition, Properties and Behaviour, Oceanography series S330 (Elsevier Science, Oxford, 1995), http://www.sciencedirect.com /science/book/9780750637152.

[55] M. J. Buckingham, Applied Acoustics 51, 225 (1997).

[56] B. Levin and M. Nosov, Physics of Tsunamis (Springer, New York, 2008).

[57] C. Salem, A. Mangeney, S. D. Bale, and P. Veltri, Astrophys. J. 702, 537 (2009).

[58] D. H. Peregrine, J. Fluid Mech. 25, 321 (1966).

[59] T. B. Benjamin, J. L. Bona, and J. J. Mahony, Philos. Trans. R. Soc. London, Ser. A 272, 47 (1972).

[60] K. He and A. Salat, Plasma Phys. Controlled Fusion 31, 123 (1989).

[61] E. L. Rempel and A. C.-L. Chian, Phys. Rev. Lett. 98, 014101 (2007).

[62] A. C.-L. Chian, R. A. Miranda, E. L. Rempel, Y. Saiki, and M. Yamada, Phys. Rev. Lett. 104, 254102 (2010).

[63] K. He, Phys. Rev. Lett. 80, 696 (1998).

[64] K. He and A. C.-L. Chian, Phys. Rev. Lett. 91, 034102 (2003).

[65] K. He and A. C.-L. Chian, Phys. Rev. E 69, 026207 (2004).

[66] V. V. Krasnoselskikh, Zh. Eksp. Teor. Fiz. 89, 498 (1985) [Sov. Phys. JETP 62(2), 282 (1985)].

[67] B. B. Mandelbrot, The Fractal Geometry of Nature, updated and augmented edition (W. H. Freeman, New York, 1983). 